\title{
MicroRNAs Silence Gene Expression by Repressing Protein Expression and/or by Promoting mRNA Decay
}

\author{
I. Behm-Ansmant, J. ReHWinkel, AND E. IZAURRALDE \\ MPI for Developmental Biology, D-72076 Tuebingen, Germany
}

\begin{abstract}
MicroRNAs (miRNAs) represent a novel class of genome-encoded eukaryotic regulatory RNAs that silence gene expression posttranscriptionally. Although the proteins mediating miRNA biogenesis and function have been identified, the precise mechanism by which miRNAs regulate the expression of target mRNAs remains unclear. We summarize recent work from our laboratory demonstrating that miRNAs silence gene expression by at least two independent mechanisms: by repressing translation and/or by promoting mRNA degradation. In Drosophila, both mechanisms require Argonaute 1 (AGO1) and the P-body component GW182. Moreover, mRNA degradation by miRNAs is effected by the enzymes involved in general mRNA decay, including deadenylases and decapping enzymes, which also localize to $P$ bodies. Our findings suggest a model for miRNA function in which AGO1 associates with miRNA targets through miRNA:mRNA base-pairing interactions. GW182 interacts with AGO1 and recruits deadenylases and decapping enzymes, leading to mRNA degradation. However, not all miRNA targets are degraded: Some stay in a translationally silent state, from which they may eventually be released. We propose that the final outcome of miRNA regulation (i.e., degradation vs. translational repression) is influenced by other RNA-binding proteins interacting with the targeted mRNA.
\end{abstract}

miRNAs represent a specific class of genome-encoded small RNAs that regulate gene expression posttranscriptionally (Bartel 2004; Filipowicz 2005). Hundreds of miRNAs and their targets have been identified or predicted in different organisms. They affect a broad range of biological processes including cell differentiation and proliferation, apoptosis, metabolism, and development (Bartel 2004; Filipowicz 2005).

miRNA regulatory functions are effected by the Argonaute proteins that promote decay or translational repression of mRNAs having sequences complementary to the miRNAs (Bartel 2004; Filipowicz 2005). Plant miRNAs are generally fully complementary to their targets and elicit endonucleolytic cleavage in the region of the mRNA that is base-paired with the miRNA. Endonucleolytic cleavage may also occur even when miRNAs are not fully complementary to their binding sites (Llave et al. 2002; Mallory et al. 2004; Allen et al. 2005; Guo et al. 2005; Schwab et al. 2005). This implies that plant miRNAs are similar to small interfering RNAs (siRNAs) with respect to their mode of action, although they differ on their mode of biogenesis (Bartel 2004; Filipowicz 2005).

The majority of animal miRNAs are only partially complementary to their targets and silence gene expression by mechanisms that remain elusive (Filipowicz 2005). At first, animal miRNAs were reported to repress translation without affecting mRNA levels (Lee et al. 1993; Wightman et al. 1993). More recently, several reports have shown that animal miRNAs can also induce significant degradation of target mRNAs despite imperfect mRNA-miRNA base-pairing (Bagga et al. 2005; Jing et al. 2005; Lim et al. 2005; Behm-Ansmant et al. 2006; Giraldez et al. 2006; Rehwinkel et al. 2006; Wu et al. 2006). Consistently, transcripts that are up-regulated in cells in which the miRNA pathway is inhibited (e.g., by depletion of Dicer or Argonaute proteins) are enriched in predicted and validated miRNA targets (Giraldez et al. 2006; Rehwinkel et al. 2006). Conversely, ectopic expression of specific miRNAs in cells in which they are not normally present leads to a reduction of the levels of transcripts containing binding sites for the miRNA (Lim et al. 2005). Finally, microarrays have been used to identify miRNA targets, indicating that miRNAs do indeed affect mRNA levels (Lim et al. 2005; Giraldez et al. 2006, Rehwinkel et al. 2006). In contrast to the situation in plants, however, under most circumstances, mRNA decay by miRNAs in animal cells may not occur via endonucleolytic cleavage but rather by directing mRNAs to the general mRNA degradation machinery, and thus by accelerating their decay (Behm-Ansmant et al. 2006; Giraldez et al. 2006; Wu et al. 2006).

Several lines of evidence support the existence of a link between the miRNA pathway and general mRNA decay. First, mammalian Argonaute proteins (AGO1-AGO4), miRNAs, and miRNA targets colocalize to cytoplasmic foci known as processing bodies (P bodies) (Jakymiw et al. 2005; Liu et al. 2005a,b; Meister et al. 2005; Pillai et al. 2005; Sen and Blau 2005). P bodies are discrete cytoplasmic domains where proteins required for bulk mRNA degradation in the $5^{\prime}$ to $3^{\prime}$ direction accumulate (e.g., deadenylases, the decapping DCP1:DCP2 complex, and the 5' to 3' exonuclease XRN1; for review, see ValenciaSanchez et al. 2006). Second, human AGO1 and AGO2 associate with the decapping coactivator DCP1 and with GW182, a protein with glycine-tryptophan repeats (GW repeats) which localizes to $\mathrm{P}$ bodies and is required for $\mathrm{P}$ body integrity (Eystathioy et al. 2002, 2003; Jakymiw et al. 2005; Liu et al. 2005a,b; Meister et al. 2005; Sen and Blau 2005). Third, depletion of GW182 in human cells impairs both miRNA function and mRNA decay triggered by complementary siRNAs (Jakymiw et al. 2005; 
Liu et al. 2005b; Meister et al. 2005). Similarly, miRNA function is impaired in Drosophila Schneider cells (S2 cells) depleted of GW182 or the decapping DCP1:DCP2 complex (Rehwinkel et al. 2005). Finally, the Caenorhabditis elegans protein AIN-1, which is related to GW182, is required for gene regulation by at least a subset of miRNAs (Ding et al. 2005).

Nevertheless, despite this link between miRNA-mediated silencing and mRNA degradation, there are multiple examples of miRNA targets for which translational repression is observed without detectable changes in the mRNA level (for review, see Valencia-Sanchez et al. 2006), suggesting that for these targets, regulation by miRNAs may occur independently of mRNA decay. How miRNAs regulate translation is not well understood. Two independent studies have shown that miRNAs inhibit translation initiation at an early stage involving the cap structure, as mRNAs translated via cap-independent mechanisms were shown to escape miRNA-mediated silencing (Humphreys et al. 2005; Pillai et al. 2005). Other studies have suggested that translation inhibition occurs after initiation on the basis of the observation that miRNAs and some targets remain associated with polysomes (Olsen and Ambros 1999; Kim et al. 2004; Nelson et al. 2004; Petersen et al. 2006). Moreover, Petersen et al. (2006) reported that mRNAs translated via cap-independent mechanisms are subjected to miRNA regulation. A possible explanation for these contradictory results is that miRNAs regulate gene expression by different mechanisms, although the possibility that these discrepancies are due to differences in experimental approaches cannot be excluded (see Valencia-Sanchez et al. 2006).

We have investigated the mechanism by which miRNAs silence gene expression. Here, we describe briefly the assays we use to monitor miRNA function in Drosophila cells, a summary of the overall results, and our current model regarding miRNA function.

\section{MIRNA-MEDIATED GENE SILENCING INVOLVES TRANSLATIONAL REPRESSION AND/OR MRNA DEGRADATION}

To investigate miRNA function in Drosophila cells, we have generated a series of reporters in which the coding region of firefly luciferase (F-Luc) is flanked by the 3'UTRs (untranslated regions) of the Drosophila gene CG10011 regulated by miR-12, and of Nerfin or Vha681 genes, which contain binding sites for miR-9b (Fig. 1a). Drosophila S2 cells are transiently transfected with the FLuc reporters, a plasmid expressing the primary miRNA transcript or the corresponding vector without insert (empty vector), and a plasmid encoding Renilla luciferase (R-Luc) as a transfection control. F-Luc activity is normalized to that of Renilla to compensate for possible differences in transfection efficiencies.

Using this assay, we have shown that expression of the F-Luc reporter having the 3'UTR of CG10011 (F-LucCG10011) is strongly reduced by cotransfection of a plasmid expressing miR-12, whereas expression of the firefly luciferase reporters fused to the Nerfin or Vha68-1 3'UTRs (F-Luc-Nerfin, F-Luc-Vha68-1) is inhibited by cotransfection of miR-9b (Fig. 1b) (Rehwinkel et al. 2005,
2006; Behm-Ansmant et al. 2006). In all three cases, silencing of luciferase expression by the cognate miRNAs is prevented in cells depleted of AGO1 (see Fig. 2) (Rehwinkel et al. 2005, 2006; Behm-Ansmant et al. 2006), demonstrating that silencing of these reporters occurs via the miRNA pathway. Note that the genomes of multicellular organisms encode multiple Argonaute proteins. In Drosophila, Argonaute paralogs have evolved specialized roles: AGO1 mediates miRNA function, whereas AGO2 catalyzes siRNA-guided endonucleolytic cleavage of mRNAs (RNA interference [RNAi]) (Okamura et al. 2004; Rand et al. 2004; Miyoshi et al. 2005).

To determine whether miRNAs silence F-Luc expression by inhibiting translation directly, or indirectly by reducing mRNA levels, we analyzed the steady-state levels of the F-Luc mRNA by northern blot and normalized them to the levels of the control R-Luc mRNA. We observed that miRNAs triggered a reduction in mRNA levels to different extents (Behm-Ansmant et al. 2006). Expression of miR-9b led to a slight decrease of the FLuc-Nerfin mRNA levels but to a significant reduction of the F-Luc-Vha68-1 mRNA levels; miR-12 triggered a strong reduction of F-Luc-CG10011 mRNA levels (Behm-Ansmant et al. 2006).

The decrease in mRNA levels observed for the Nerfin and Vha68-1 reporters is significantly smaller than the reduction observed in F-Luc activity. Indeed, after normalizing firefly luciferase activity to the corresponding mRNA levels, we observed that miR-9b leads to a net 5fold and 2.5-fold reduction in protein expression for the Nerfin and Vha68-1 reporters, respectively (Fig. 1c). In contrast, silencing of F-Luc-CG10011 by miR-12 can be attributed primarily to the reduction of mRNA levels (Fig. 1c). These results indicate that miRNAs silence gene expression by two mechanisms, one involving inhibition of protein expression and one a reduction of mRNA levels.

\section{MIRNAS ACCELERATE MRNA DEGRADATION}

To investigate whether the reduction of mRNA levels caused by miRNAs is a consequence of increased mRNA degradation, we measured the half-lives of the reporters in the absence or presence of the cognate miRNAs following inhibition of transcription with actinomycin D. The halflives of the F-Luc reporter having the Nerfin, Vha68-1, and CG10011 3'UTRs were about 141 minutes, 200 minutes, and longer than 300 minutes, respectively. In cells expressing the miRNAs, the half-lives of these reporters were reduced to about 38 minutes, 23 minutes, and 10 minutes (Fig. 1d). Thus, even for the Nerfin reporter, whose steady-state levels do not change dramatically in the presence of miR-9b, the degradation rate is increased.

In the presence of the miRNAs, the mRNA reporters have biphasic decay kinetics, suggesting the existence of a heterogeneous pool of mRNAs (for instance, free or bound to the miRNA) undergoing different rates of degradation. In addition, the reduction in half-lives is more marked than the changes in steady-state mRNA levels. One possible explanation for this discrepancy is that steady-state mRNA levels are determined by transcription and decay rates, and at steady state, transcription rates may compensate for decay. Alternatively, we cannot 
a MiRNA reporters



\section{b Luciferase activity \\ $\square$ empty $\square+$ miRNA}



C Net effect on luciferase activity empty $\square$ + miRNA



\section{d Reporter half-lives}


Figure 1. Silencing by miRNAs is effected by changes in protein expression and/or mRNA stability. (a) miRNA reporters. The F-Luc open reading frame (F-Luc) is flanked by the 3'UTRs of Nerfin, Vha68-1, or CG10011 mRNAs, which contain binding sites for miR$9 \mathrm{~b}$ or miR-12 as indicated. $(b, c)$ S2 cells were transfected with plasmids expressing miRNA reporters (Nerfin, Vha68-1, or CG10011), plasmids expressing miR-9b or miR-12 (red bars), or the corresponding empty vector (black bars). Renilla luciferase (R-Luc) served as a transfection control. F-Luc activity and the corresponding mRNA levels were measured and normalized to those of the Renilla. Normalized F-Luc activities in cells transfected with the empty vector (black bars) were set to $100 \%$. In panel $c$, the normalized values of F-Luc activity shown in panel $b$ are divided by the normalized mRNA levels (not shown) to estimate the net effect of miRNAs on protein expression. Error bars represent standard deviations from at least three independent experiments. $(d)$ S2 cells were transfected with the miRNA reporters shown in panel $a$. Three days after transfection, cells were treated with actinomycin D and harvested at the indicated time points. The decay of the F-Luc reporters was monitored in cells coexpressing the miRNA (pink) or the corresponding empty vector (blue). RNA samples were analyzed by northern blot (not shown). The levels of F-Luc reporter transcripts normalized to those of the long-lived rp49 mRNA are plotted as a function of time. mRNA half-lives $\left(t_{1 / 2}\right)$ are indicated.

exclude the possibility that the actinomycin D treatment is having secondary effects on mRNA stability.

\section{MIRNAS PROMOTE DEADENYLATION AND DECAPPING}

We recently reported that mRNA decay by miRNAs requires components of the CCR4:NOT deadenylase complex and the decapping DCP1:DCP2 complex (Behm-Ansmant et al. 2006). Figure 2a,b shows a comparison of the effects of depleting components of the deadenylase or decapping complexes on miRNA activity for the Nerfin and CG10011 reporters, which are regulated mainly at the translational level and at the mRNA levels, respectively. In this representation, F-Luc activity or mRNA levels are normalized to those of Renilla. For each knockdown, the normalized F-Luc activities or mRNA levels measured in the absence of the miRNA are set to 100 (not shown).

For the Nerfin reporter, expression of miR-9b strongly reduces F-Luc activity but mRNA levels only slightly (Fig. 2a, black and orange bars, respectively). Both luciferase expression and mRNA levels are restored in cells depleted of AGO1 or GW182. This indicates that 
a Nerfin + miR-9b

Normalized F-Luc activity $\square$ Normalized F-Luc mRNA levels



\section{b $\quad$ G $10011+m i R 12$}

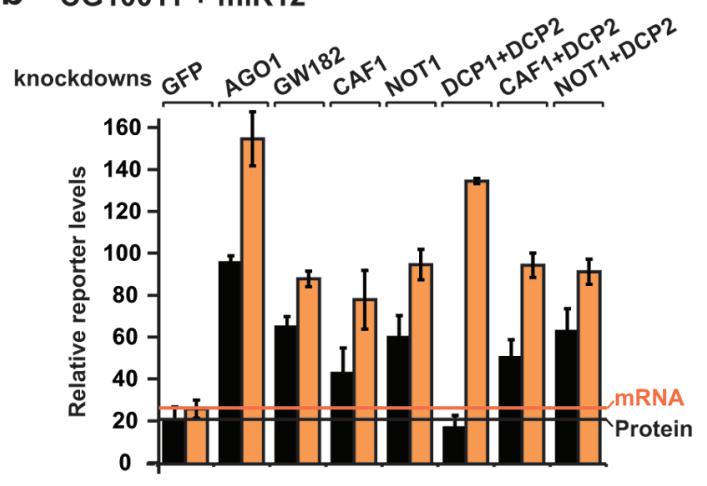

C CG1998 mRNA (K-box miRNAs)

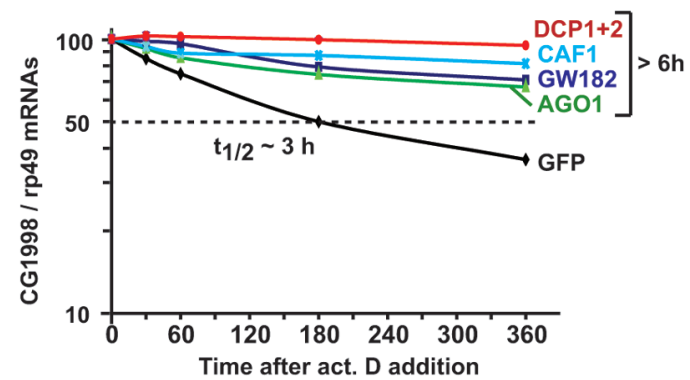

Figure 2. mRNA decay triggered by miRNAs occurs by a deadenylation and decapping mechanism requiring GW182. $(a, b) \mathrm{S} 2$ cells depleted of the proteins indicated above the panels were transfected with plasmids expressing the Nerfin or CG10011 miRNA reporters. Control cells were treated with green fluorescent protein (GFP) double-stranded RNA (dsRNA). F-Luc activity and the corresponding mRNA levels were measured and normalized to those of the Renilla. Normalized F-Luc activities (black bars) and mRNA levels (orange bars) in the presence of the miRNA are shown. In cells transfected with the empty vector, these values were set to $100 \%$ for each knockdown (not shown). (Black horizontal lines) F-Luc activity in control cells; (orange horizontal lines) mRNA levels in control cells. Error bars represent standard deviations from three independent experiments. (c) Endogenous miRNA targets are degraded by deadenylation and decapping. The decay of CG1998 mRNA (a predicted target of K-box miRNAs) was monitored in S2 cells depleted of AGO1, GW182, CAF1, or DCP1:DCP2 following inhibition of transcription by actinomycin D. Control cells were treated with GFP dsRNA. The levels of the CG1998 mRNA were analyzed by northern blot, normalized to rp49 mRNA (not shown), and plotted against time. mRNA half-lives $\left(t_{1 / 2}\right)$ calculated from the decay curves are indicated.
GW182 also has a role in translational silencing by miRNAs, in agreement with previous studies (Jakymiw et al. 2005; Liu et al. 2005b; Meister et al. 2005; Rehwinkel et al. 2005).

There are two major deadenylase complexes in Drosophila: the PAN2:PAN3 and the CCR4:NOT complexes (Temme et al. 2004). Depletion of PAN2, PAN3, CAF1, or NOT1 does not have a significant effect on luciferase expression or mRNA levels of the Nerfin reporter (Fig. 2a) (data not shown). Similarly, codepletion of CAF1 or NOT1 with DCP2 does not restore luciferase expression or mRNA levels (Fig. 2a). In contrast, depletion of DCP1:DCP2 restores mRNA levels indicating that this reporter is degraded by decapping (Fig. 2a). Although mRNA levels are restored in cells depleted of decapping enzymes, luciferase expression is not. A potential explanation for this lack of restoration is provided by the analysis of mRNA levels by northern blotting. Indeed, we observe that reporter transcripts accumulating in cells depleted of the decapping enzymes are deadenylated and are thus expected to be translated less efficiently (BehmAnsmant et al. 2006). Deadenylation is observed only in the presence of the miRNA, indicating that miRNAs trigger deadenylation. Deadenylation is, however, unlikely to be the cause of the translational repression because luciferase activity is not restored in cells depleted of deadenylases.

When we analyzed the CG10011 reporter, which is mainly regulated at the mRNA level, we observed that depletion of AGO1 or GW182 restores both luciferase expression and mRNA levels, indicating that mRNA decay is AGO1- and GW182-dependent. Depletion of CAF1 or NOT1 individually or in combination with DCP2 also restores both luciferase expression and mRNA levels, providing strong evidence for the conclusion that regulation of this reporter by miR-12 occurs at the level of mRNA degradation (Fig. 2b). Again, depletion of DCP1:DCP2 restores mRNA levels, but as observed for the Nerfin reporter, transcripts accumulating in these cells are deadenylated in the presence of the miRNA (BehmAnsmant et al. 2006), providing a possible explanation for the partial or lack of restoration of luciferase expression.

In summary, these findings indicate that gene silencing by miRNAs involves translational repression and/or mRNA deadenylation and decapping and both require GW182. The results from our laboratory in Drosophila cells are remarkably consistent with recent studies in zebra fish embryos and human cells showing that miRNAs promote accelerated deadenylation of their targets (Giraldez et al. 2006; Wu et al. 2006). Moreover, Wu et al. (2006) reported that mRNAs lacking a poly(A) tail are nevertheless subjected to translational repression by miRNAs, indicating that this repression occurs by a deadenylation-independent mechanism.

A question that remains open is whether miRNA-mediated mRNA degradation is a consequence of translational repression or whether these represent two independent mechanisms by which miRNAs silence gene expression as proposed by $\mathrm{Wu}$ et al. (2006). As mentioned above, inhibition of translation is not always accompanied by changes in mRNA levels. Conversely, targets regulated 
mainly at the translational level are subjected to accelerated deadenylation, but depletion of CAF1 or NOT1 does not restore protein expression. These findings suggest that translational repression and mRNA degradation represent two independent mechanisms by which miRNAs regulate gene expression.

\section{ENDOGENOUS MIRNA REPORTERS ARE DEGRADED IN AN AGO1- AND GW182-DEPENDENT MANNER}

To investigate the significance of the results obtained with the reporters for the miRNA pathway, we analyzed mRNA expression profiles in S2 cells depleted of GW182 or AGO1. We observed that depletion of these proteins leads to correlated changes in mRNA expression levels, indicating that they act in the same pathway. Furthermore, transcripts commonly up-regulated by AGO1 and GW182 are enriched in predicted and validated miRNA targets. This shows that, as for luciferase reporters, endogenous miRNA targets are regulated by GW182 (Behm-Ansmant et al. 2006).

We also analyzed the degradation rate of endogenous miRNA targets identified by expression profiling of AGO1 or GW182-depleted cells (Behm-Ansmant et al. 2006; Rehwinkel et al. 2006). The Axs and CG1998 mRNAs are predicted targets of miR-285 and K-box miRNAs, respectively (Stark et al. 2005). These mRNAs are at least three- to sixfold up-regulated in cells depleted of AGO1 or GW182. In cells depleted of AGO1, GW182, CAF1, or DCP1:DCP2, the half-lives of these mRNAs increase from approximately 3 hours to more than 6 hours, suggesting that they are degraded by deadenylation and decapping in an AGO1- and GW182-dependent manner (Fig. 2c) (Behm-Ansmant et al. 2006).

It is worth noting that for some endogenous miRNA targets, depletion of decapping enzymes or of components of the CCR4:NOT complex leads to a stronger stabilization of the mRNA than that observed in cells depleted of AGO1 or GW182. This indicates that only a fraction of these endogenous transcripts is targeted by the cognate miRNAs. Depletion of the CCR4:NOT complex or of the decapping enzymes prevents general degradation and degradation via the miRNA pathway, leading to a stronger stabilization of the transcripts. This is likely to be the case for mRNAs targeted by low-abundance miRNAs.

Together, the results obtained for the F-Luc reporters and endogenous targets demonstrate unequivocally a link between miRNA-mediated gene silencing and the machinery for general mRNA degradation. They also show that GW182 is a critical effector of miRNA function in animal cells.

\section{GW182 BELONGS TO A CONSERVED FAMILY OF PROTEINS}

GW182 belongs to a family of proteins containing a central ubiquitin-associated (UBA) domain, a carboxy-terminal RNA recognition motif (RRM), and three blocks of glycine-tryptophan repeats (referred to as amino-terminal, middle, and carboxy-terminal GW repeats). Furthermore, a glutamine-rich (Q-rich) region is located between the UBA domain and the RRM (Fig. 3a) (Eystathioy et al. 2002; Behm-Ansmant et al. 2006).

Vertebrates contain three GW182 family members (TNRC6A/GW182, TNRC6B, and TNRC6C), but there is a single ortholog in insects and no ortholog in worms or fungi. Sequence alignment of all members of the protein family revealed the presence of two highly conserved motifs (I and II) of approximately 50 residues within the amino-terminal GW repeats (Fig. 3a). The insect proteins start exactly with motif I, whereas the vertebrate orthologs have amino-terminal extensions. Interestingly, the amino-terminal domain of GW182 proteins encompassing motif II bears similarity to the GW-like regions in

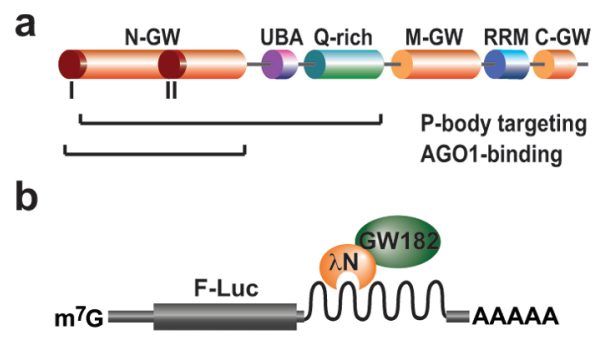

\section{F-Luc-5BoxB + $\lambda$ N-GW182}

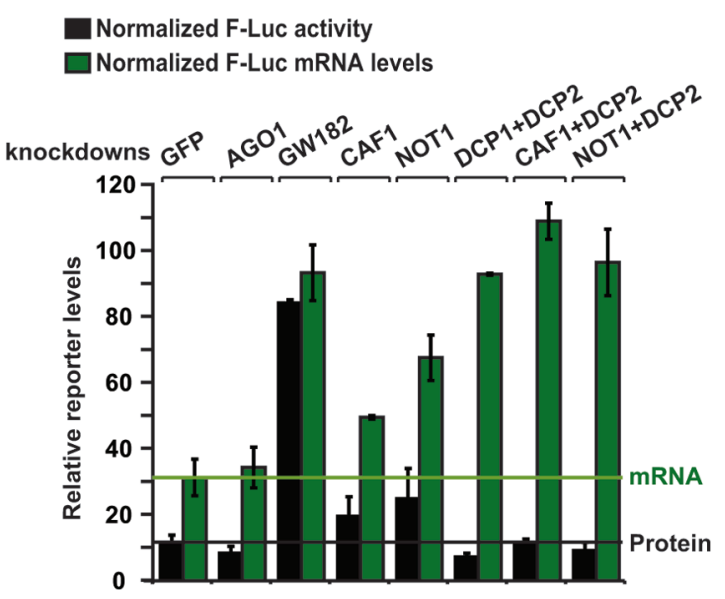

Figure 3. GW182 acts downstream from AGO1 in the miRNA pathway. (a) Domain organization of Drosophila GW182. (NGW, M-GW, and C-GW) Amino-terminal, middle, and carboxy-terminal GW repeats, respectively; (UBA) ubiquitinassociated domain; (Q-rich) region rich in glutamine; (RRM) RNA recognition motif. Motifs I and II are indicated. The protein domains sufficient for the localization to $\mathrm{P}$ bodies and the interaction with $\mathrm{AGO} 1$ are shown below the protein outline. (b) Schematic representation of the F-Luc-5BoxB tethering reporter. (c) S2 cells depleted of the proteins indicated above the panel were transfected with the F-Luc-5BoxB reporter, a plasmid expressing Renilla luciferase, and vectors expressing the $\lambda \mathrm{N}$-peptide or $\lambda \mathrm{N}-\mathrm{GW} 182$. F-Luc activities (black bars) and mRNA levels (green bars) were quantitated in three independent experiments and normalized to that of the Renilla control. In cells expressing the $\lambda \mathrm{N}$ peptide alone, these values were set to 100 for each knockdown (not shown). (Black horizontal lines) Normalized F-Luc activity; (green horizontal lines) mRNA levels in control cells. Mean values plus or minus standard deviations from three independent experiments are shown. 
the C. elegans protein AIN-1, involved in the miRNA pathway (Ding et al. 2005). However, AIN-1 contains no UBA, Q-rich, or RRM domains, suggesting that it represents a functional analog.

C. elegans AIN-1, Drosophila GW182, and human TNRC6A/GW182 and TNRC6B localize to P bodies and interact with Argonaute proteins (Ding et al. 2005; Jakymiw et al. 2005; Liu et al. 2005a,b; Meister et al. 2005; Sen and Blau 2005; Behm-Ansmant et al. 2006). We have shown that it is the amino-terminal GW-repeat domain of Drosophila GW182 that mediates the interaction with the PIWI domain of AGO1 (Fig. 3a) (BehmAnsmant et al. 2006). These findings suggest a conserved role for these repeats, and most likely for the highly conserved motif II, in mediating the interaction with Argonaute proteins. Interestingly, the PIWI domain adopts an RNase-H-like fold and is catalytically active, at least for a subset of Argonaute proteins including Drosophila AGO1 (for review, see Lingel and Sattler 2005; Miyoshi et al. 2005). It would therefore be of interest to determine whether the amino-terminal GW repeats of GW182 can modulate the catalytic activity of this domain.

Apart from the interaction with AGO1, the amino-terminal repeats and the UBA and Q-rich domains contribute to the localization of GW182 in P bodies (Fig. 3a) (Behm-Ansmant et al. 2006), which is in turn required for P-body integrity (Eystathioy et al. 2002, 2003). This suggests that GW182 may act as a molecular scaffold bringing together AGO1-containing RISCs (RNA-induced silencing complex) and mRNA decay enzymes, possibly nucleating the assembly of $\mathrm{P}$ bodies.

\section{GW182 ACTS DOWNSTREAM FROM AGO1}

The analyses described above combined with previous studies indicate that GW182 is recruited to miRNA targets via interactions with the Argonaute proteins (Jakymiw et al. 2005; Liu et al. 2005b; Meister et al. 2005; Behm-Ansmant et al. 2006). We used a tethering assay to begin to investigate the consequences of the recruitment of GW182 to miRNA targets. In this assay, GW182 is expressed as a fusion with the $\lambda \mathrm{N}$ peptide, which binds with high affinity to five hairpins (BoxB sites) present in the 3'UTR of a luciferase reporter mRNA (F-Luc-5BoxB reporter, Fig. 3b). In this way, GW182 is directly tethered to the 3'UTR of the reporter, bypassing the requirement for AGO1 or miRNAs.

Using this assay, we have shown that tethering of GW182 to the F-Luc-5BoxB reporter silences its expression and promotes mRNA degradation (Fig. 3c) (BehmAnsmant et al. 2006). The decrease in mRNA levels observed for the tethered mRNA does not, however, fully account for the strong reduction in F-Luc activity (Fig. $3 c$ ), indicating that GW182 silences expression of bound transcripts by a dual mechanism involving inhibition of protein expression and reduction of mRNA levels. As with miRNAs, mRNA degradation by GW182 requires the CCR4:NOT and the DCP1:DCP2 complexes. Indeed, the levels of a reporter transcript tethered to GW182 are restored in cells depleted of CAF1, NOT1, or the decapping enzymes. The restoration of mRNA levels in cells depleted of CAF1 or NOT1 is not accompanied by a proportional increase in F-Luc expression (Fig. 3c), providing further evidence for a role of GW182 in translational regulation. Restoration of mRNA levels, but not of luciferase activity, is also observed in cells co-depleted of CAF1 and DCP2 or NOT1 and DCP2, demonstrating that GW182 promotes deadenylation and decapping of bound mRNAs (Fig. 3c). Thus, binding of GW182 appears to be a point of no return which marks transcripts as targets for the general mRNA degradation machinery.

Remarkably, GW182-mediated silencing and decay does not require AGO1 (Fig. 3c). This observation places GW182 downstream from AGO1 in the miRNA pathway. Several lines of evidence support this conclusion. First, endogenous and ectopically expressed GW182 localizes to $\mathrm{P}$ bodies together with decapping enzymes and the deadenylase complex (Eystathioy et al. 2002, 2003). Second, GW182 interacts with AGO1 and recruits AGO1 to $\mathrm{P}$ bodies when the two proteins are coexpressed (Jakymiw et al. 2005; Liu et al. 2005a,b; Meister et al. 2005; Sen and Blau 2005; Behm-Ansmant et al. 2006). Third, as mentioned above, degradation of mRNAs by GW182 or miRNAs requires the CCR4:NOT and DCP1:DCP2 complexes (Behm-Ansmant et al. 2006). Finally, miRNAs repress translation and promote mRNA degradation in a GW182-dependent manner (Jakymiw et al. 2005; Liu et al. 2005b; Meister et al. 2005; Rehwinkel et al. 2005; Behm-Ansmant et al. 2006).

\section{CONCLUSIONS}

On the basis of the results described above and the observations that GW182 associates with AGO1 and is required for miRNA-mediated gene silencing (Ding et al. 2005; Jakymiw et al. 2005; Liu et al. 2005b; Meister et al. 2005; Rehwinkel et al. 2005; Behm-Ansmant et al. 2006), we propose the following model for the mechanism of miRNA-mediated gene silencing (Fig. 4): AGO1 binds miRNA targets by means of miRNA:mRNA base-pairing interactions. AGO1 may then recruit GW182 and both proteins repress translations (Fig. 4). GW182 also marks



Figure 4. Model for miRNA-mediated gene silencing. miRNAmediated gene silencing involves multiple mechanisms including translational repression, deadenylation, and decapping. In Drosophila, these mechanisms require AGO1 and the P-body protein GW182. Deadenylation and decapping are catalyzed by the DCP1:DCP2 decapping complex and the CCR4:NOT deadenylase complex, respectively. The mechanism by which miRNAs repress translation remains to be established. In addition, it is unclear whether silencing occurs in P bodies, although all proteins shown in this diagram have been localized to $\mathrm{P}$ bodies. 
the transcripts as targets for decay via a deadenylation and decapping mechanism. The contribution of translational repression or mRNA degradation to gene silencing appears to differ for each miRNA:target pair and is likely to depend on the particular set of proteins bound to the 3'UTR of the mRNA. Furthermore, binding of specific RNA-binding proteins to the 3'UTR of miRNA targets can revert miRNA-mediated silencing under some physiological conditions (Bhattacharyya et al. 2006). A major challenge for future studies will be to identify how specific RNA-binding proteins influence the final outcome of miRNA regulation. Another important question that remains open is the mechanism by which miRNAs repress translation. Finally, whether silencing by miRNAs occurs in the cytosol or in cytoplasmic domains such as P bodies is currently a matter of debate that requires further investigation and a deeper understanding of the assembly, composition, and cellular function of these bodies.

\section{ACKNOWLEDGMENTS}

We are grateful to D.J. Thomas for comments on the manuscript. This study was supported by EMBO and the Human Frontier Science Program Organization (HFSPO). I.B.-A. is the recipient of a fellowship from EMBO.

\section{REFERENCES}

Allen E., Xie Z., Gustafson A.M., and Carrington J.C. 2005. microRNA-directed phasing during trans-acting siRNA biogenesis in plants. Cell 121: 207.

Bagga S., Bracht J., Hunter S., Massirer K., Holtz J., Eachus R., and Pasquinelli A.E. 2005. Regulation by let-7 and lin-4 miRNAs results in target mRNA degradation. Cell 122: 553.

Bartel D.P. 2004. MicroRNAs: Genomics, biogenesis, mechanism, and function. Cell 116: 281.

Behm-Ansmant I., Rehwinkel J., Doerks T., Stark A., Bork P., and Izaurralde E. 2006. mRNA degradation by miRNAs and GW182 requires both CCR4:NOT deadenylase and DCP1:DCP2 decapping complexes. Genes Dev. 20: 1885.

Bhattacharyya S.N., Habermacher R., Martine U., Closs E.I., and Filipowicz W. 2006. Relief of microRNA-mediated translational repression in human cells subjected to stress. Cell 125: 1111.

Ding L., Spencer A., Morita K., and Han M. 2005. The developmental timing regulator AIN-1 interacts with miRISCs and may target the argonaute protein ALG-1 to cytoplasmic P bodies in C. elegans. Mol. Cell 19: 437.

Eystathioy T., Chan E.K., Tenenbaum S.A., Keene J.D., Griffith K., and Fritzler M.J. 2002. A phosphorylated cytoplasmic autoantigen, GW182, associates with a unique population of human mRNAs within novel cytoplasmic speckles. Mol. Biol. Cell 13: 1338 .

Eystathioy T., Jakymiw A., Chan E.K., Seraphin B., Cougot N., and Fritzler M.J. 2003. The GW182 protein colocalizes with mRNA degradation associated proteins hDcp 1 and hLSm4 in cytoplasmic GW-bodies. RNA 9: 1171.

Filipowicz W. 2005. RNAi: The nuts and bolts of the RISC machine. Cell 122: 17.

Giraldez A.J., Mishima Y., Rihel J., Grocock R.J., Van Dongen S., Inoue K., Enright A.J., and Schier A.F. 2006. Zebrafish MiR-430 promotes deadenylation and clearance of maternal mRNAs. Science 312: 75.

Guo H.S., Xie Q., Fei J.F., and Chua N.H. 2005. MicroRNA directs mRNA cleavage of the transcription factor NAC1 to downregulate auxin signals for Arabidopsis lateral root development. Plant Cell 17: 1376
Humphreys D.T., Westman B.J., Martin D.I., and Preiss T. 2005. MicroRNAs control translation initiation by inhibiting eukaryotic initiation factor 4E/cap and poly(A) tail function. Proc. Natl. Acad. Sci. 102: 16961.

Jakymiw A., Lian S., Eystathioy T., Li S., Satoh M., Hamel J.C., Fritzler M.J., and Chan E.K. 2005. Disruption of GW bodies impairs mammalian RNA interference. Nat. Cell Biol. 7: 1167.

Jing Q., Huang S., Guth S., Zarubin T., Motoyama A., Chen J., Di Padova F., Lin S.C., Gram H., and Han J. 2005. Involvement of microRNA in AU-rich element-mediated mRNA instability. Cell 120: 623.

Kim J., Krichevsky A., Grad Y., Hayes G.D., Kosik K.S., Church G.M., and Ruvkun G. 2004. Identification of many microRNAs that copurify with polyribosomes in mammalian neurons. Proc. Natl. Acad. Sci. 101: 360.

Lee R.C., Feinbaum R.L., and Ambros V. 1993. The C. elegans heterochronic gene lin-4 encodes small RNAs with antisense complementarity to lin-14. Cell 75: 843 .

Lim L.P., Lau N.C., Garrett-Engele P., Grimson A., Schelter J.M., Castle J., Bartel D.P., Linsley P.S., and Johnson J.M. 2005. Microarray analysis shows that some microRNAs downregulate large numbers of target mRNAs. Nature 433: 769.

Lingel A. and Sattler M. 2005. Novel modes of protein-RNA recognition in the RNAi pathway. Curr. Opin. Struct. Biol. 15: 107.

Llave C., Xie Z., Kasschau K.D., and Carrington J.C. 2002. Cleavage of Scarecrow-like mRNA targets directed by a class of Arabidopsis miRNA. Science 297: 2053.

Liu J., Valencia-Sanchez M.A., Hannon G.J., and Parker R. 2005a. MicroRNA-dependent localization of targeted mRNAs to mammalian P-bodies. Nat. Cell Biol. 7: 719.

Liu J., Rivas F.V., Wohlschlegel J., Yates J.R., Parker R., and Hannon G.J. 2005b. A role for the P-body component GW182 in microRNA function. Nat. Cell Biol. 7: 1261.

Mallory A.C., Reinhart B.J., Jones-Rhoades M.W., Tang G., Zamore P.D., Barton M.K., and Bartel D.P. 2004. MicroRNA control of PHABULOSA in leaf development: Importance of pairing to the microRNA 5 ' region. EMBO J. 23: 3356.

Meister G., Landthaler M., Peters L., Chen P.Y., Urlaub H., Luhrmann R., and Tuschl T. 2005. Identification of novel Argonaute-associated proteins. Curr. Biol. 15: 2149.

Miyoshi K., Tsukumo H., Nagami T., Siomi H., and Siomi M.C. 2005. Slicer function of Drosophila Argonautes and its involvement in RISC formation. Genes Dev. 19: 2837.

Nelson P.T., Hatzigeorgiou A.G., and Mourelatos Z. 2004. miRNP:mRNA association in polyribosomes in a human neuronal cell line. $R N A$ 10: 387 .

Okamura K., Ishizuka A., Siomi H., and Siomi MC. 2004. Distinct roles for Argonaute proteins in small RNA-directed RNA cleavage pathways. Genes Dev. 18: 1655.

Olsen P.H. and Ambros V. 1999. The lin-4 regulatory RNA controls developmental timing in Caenorhabditis elegans by blocking LIN-14 protein synthesis after the initiation of translation. Dev. Biol. 216: 671 .

Petersen C.P., Bordeleau M.E., Pelletier J., and Sharp P.A. 2006. Short RNAs repress translation after initiation in mammalian cells. Mol. Cell 21: 533.

Pillai R.S., Bhattacharyya S.N., Artus C.G., Zoller T., Cougot N., Basyuk E., Bertrand E., and Filipowicz W. 2005. Inhibition of translational initiation by let-7 microRNA in human cells. Science 309: 1573.

Rand T.A., Ginalski K., Grishin N.V., and Wang X. 2004. Biochemical identification of Argonaute 2 as the sole protein required for RNA-induced silencing complex activity. Proc. Natl. Acad. Sci. 101: 14385.

Rehwinkel J., Behm-Ansmant I., Gatfield D., and Izaurralde E. 2005. A crucial role for GW182 and the DCP1:DCP2 decapping complex in miRNA-mediated gene silencing. RNA 11: 1640.

Rehwinkel J., Natalin P., Stark A., Brennecke J., Cohen S.M., and Izaurralde E. 2006. Genome-wide analysis of mRNAs regulated by Drosha and Argonaute proteins in Drosophila. Mol. Cell. Biol. 26: 2965. 
Schwab R., Palatnik J.F., Riester M., Schommer C., Schmid M., and Weigel D. 2005. Specific effects of microRNAs on the plant transcriptome. Dev. Cell 8: 517.

Sen G.L. and Blau H.M. 2005. Argonaute 2/RISC resides in sites of mammalian mRNA decay known as cytoplasmic bodies. Nat. Cell Biol. 7: 633.

Stark A., Brennecke J., Bushati N., Russell R.B., and Cohen S.M. 2005. Animal microRNAs confer robustness to gene expression and have a significant impact on 3'UTR evolution. Cell 123: 1133.

Temme C., Zaessinger S., Meyer S., Simonelig M., and Wahle
E. 2004. A complex containing the CCR4 and CAF1 proteins is involved in mRNA deadenylation in Drosophila. EMBO J. 23: 2862.

Valencia-Sanchez M.A., Liu J., Hannon G.J., and Parker R. 2006. Control of translation and mRNA degradation by miRNAs and siRNAs. Genes Dev. 20: 515.

Wightman B., Ha I., and Ruvkun G. 1993. Posttranscriptional regulation of the heterochronic gene lin-14 by lin-4 mediates temporal pattern formation in C. elegans. Cell 75: 855.

Wu L., Fan J., and Belasco J.G. 2006. MicroRNAs direct rapid deadenylation of mRNA. Proc. Natl. Acad. Sci. 103: 4034. 




\section{MicroRNAs Silence Gene Expression by Repressing Protein Expression and/or by Promoting mRNA Decay}

I. BEHM-ANSMANT, J. REHWINKEL and E. IZAURRALDE

Cold Spring Harb Symp Quant Biol 2006 71: 523-530

Access the most recent version at doi:10.1101/sqb.2006.71.013

References This article cites 39 articles, 19 of which can be accessed free at: http://symposium.cshlp.org/content/71/523.full.html\#ref-list-1

License

Email Alerting Receive free email alerts when new articles cite this article - sign up in Service the box at the top right corner of the article or click here. 